PROCEEDINGS OF THE

AMERICAN MATHEMATICAL SOCIETY

Volume 109, Number 4, August 1990

\title{
ALGEBRAS WITH LARGE HOMOLOGICAL DIMENSIONS
}

\author{
ELLEN KIRKMAN AND JAMES KUZMANOVICH
}

(Communicated by Maurice Auslander)

\begin{abstract}
An example is given of a semiprimary ring with infinite finitistic dimension. The construction shows that the global dimensions of finite dimensional algebras of finite global dimension cannot be bounded by a function of only Loewy length and the number of nonisomorphic simple modules.
\end{abstract}

The (right) finitistic global dimension $\operatorname{rFPD}(A)$ of a ring $A$ is the supremum of the projective dimensions of the right $A$-modules of finite projective dimension; we denote the supremum of the projective dimensions of the right finitely generated $A$-modules of finite projective dimension by $\operatorname{rfPD}(A)$. When $A$ is a semiprimary ring with $(\operatorname{rad} A)^{2}=0$ it is easy to show that $\operatorname{rFPD}(A)$ is finite; in this note we present a semiprimary graded $\operatorname{ring} A$ with $(\operatorname{rad} A)^{4}=0$ and $\operatorname{rfPD}(A)=\infty$. It is a long standing open question (see [B]) whether $\operatorname{rfPD}(A)$ (or $\operatorname{rFPD}(A)$ ) is finite for all finite dimensional algebras $A$; there has been some recent progress on the question (see [Z1, GKK, IZ, GZ-H]) including a proof [GZ-H] of the fact that $\operatorname{rfPD}(A)<\infty$ when $A$ is a right Artinian ring with $(\operatorname{rad} A)^{3}=0$. The example in this note shows that the Finitistic Dimension Conjecture is not true for semiprimary rings; it, of course, remains open for finite dimensional algebras and Artinian rings.

A related question of current interest is to find bounds on the global dimension of a finite dimensional algebra $A$ of finite global dimension. Schofield [S] proved that there exists an integer-valued function $f$, such that if $A$ is a finite dimensional $k$-algebra with vector space dimension $[A: k]=n$ and with finite global dimension, then $\operatorname{gldim}(A) \leq f(n)$; the nature of this function $f$ is unknown, but in all known examples of algebras with finite global dimension, the global dimension of $A$ does not exceed the vector space dimension of $A$. Examples of finite dimensional algebras of arbitrarily large finite global dimension can be produced by increasing either the number of isomorphism classes of simples or the Loewy length. It has been shown [G] that finite dimensional algebras with exactly two isomorphism classes of simple right modules can have

Received by the editors June 28, 1989 and, in revised form, October 25, 1989.

1980 Mathematics Subject Classification (1985 Revision). Primary 16A46, 16A51, 16A60.

Key words and phrases. Finitistic dimension, global dimension, semiprimary rings, finite dimensional algebras.

The first author was partially supported by the National Science Foundation DMS- 8807484 .

(c) 1990 American Mathematical Society $0002-9939 / 90 \$ 1.00+\$ .25$ per page 
arbitrarily large finite global dimension, but for certain classes of algebras, upper bounds on the global dimension of finite dimensional algebras of finite global dimension have been obtained (see [GHZ, Gu, Z1, Z2, Z-H]). Bounds on the finitistic dimension [GKK, GZ-H, Z-H], of course, are also bounds on the global dimension of algebras of finite global dimension; in [IZ] it is shown that $\operatorname{rFPD}(A)$ is bounded by $[\operatorname{rad} A: k]$ for any finite dimensional monomial algebra $A$. The example constructed in this note is a direct limit of finite dimensional algebras $A_{i}$ of finite (but increasing) global dimensions; for each of the algebras $A_{i},\left(\operatorname{rad} A_{i}\right)^{4}=0$, there are exactly two isomorphism classes of simple right $A_{i}$-modules, $\operatorname{gldim} A_{i}=2 i+1$, and $\left[\operatorname{rad} A_{i}: k\right]=2 i^{2}+i$. Hence there does not exist an upper bound on the global dimension of a finite dimensional algebra $A$ of finite global dimension which depends only upon the Loewy length of $A$ and the number of isomorphism classes of simple right $A$-modules.

Let $k$ be a field and $\Gamma$ a directed graph. The path algebra $k \Gamma$ of $\Gamma$ over $k$ is the $k$-vector space with basis consisting of the set of all paths in $\Gamma$, and with multiplication of paths given by $\alpha \cdot \beta=\alpha \beta$ if the terminal vertex of $\alpha$ is the initial vertex of $\beta$ and $\alpha \cdot \beta=0$ otherwise; the multiplication is extended bilinearly to all of $k \Gamma$.

The example $A$ with properties described above is a factor ring of the path algebra $k \Gamma$ of the quiver $\Gamma$ below with the relations indicated, for arrows $\left\{a_{i}, b_{i}: i \in \mathbb{N}\right\}$.

Quiver $\Gamma$ :

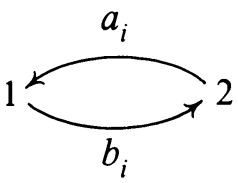

Relations $\rho: \quad b_{i} a_{j} b_{l}=0$ for all $i, j, l$,

$a_{i} b_{i+l}-a_{i+l} b_{i+l}=0$ for $l \geq 1$,

$a_{i} b_{j}=0$ for $i>j$,

$b_{i} a_{i}=0$ for all $i$.

Let $A=k \Gamma /\langle\rho\rangle$. It is not difficult to check that the following properties hold for $A$ :

1. Letting $e_{i}$ denote the idempotent associated to vertex $i$, a basis for $A$ over $k$ is: $\left\{e_{i}, a_{i}, b_{i}, a_{i} b_{i}, b_{i} a_{j}\right.$ for $i \neq j, a_{i} b_{i} a_{j}$ for $\left.i \neq j\right\}$.

2. There are exactly two isomorphism classes of simple right $A$-modules, $\operatorname{rad} A=\left\langle a_{i}, b_{i}\right\rangle,(\operatorname{rad} A)^{4}=0, A$ is a semiprimary $\operatorname{ring}$, and $A$ is graded by powers of $\operatorname{rad} A$ since the relations are homogeneous.

3. We compute right annihilators of certain elements of $A$ :

rannih $a_{1}=e_{2} A$,

rannih $b_{1}=a_{1} A \oplus e_{1} A \quad$ (note that $a_{j} b_{j}=a_{1} b_{j} \in a_{1} A$ for $j \geq 2$ ),

rannih $a_{2}=b_{1} A \oplus e_{2} A$, and

rannih $b_{2}=a_{2} A \oplus a_{1} b_{1} A \oplus e_{1} A$ and $\operatorname{rannih} a_{1} b_{1}=\operatorname{rannih} b_{1}$. 
For $i \geq 3$,

rannih $a_{i}=b_{1} A \oplus \cdots \oplus b_{i-1} A \oplus e_{2} A$,

rannih $b_{i}=a_{i} A \oplus a_{1} b_{1} A \oplus \cdots \oplus a_{i-1} b_{i-1} A \oplus e_{1} A, \quad$ and

rannih $a_{i} b_{i}=\operatorname{rannih} b_{i}$.

4. Considering the exact sequence $0 \rightarrow \operatorname{rannih} x \rightarrow A \rightarrow x A \rightarrow 0$ it follows from 3 that $\operatorname{pd}\left(a_{1} A\right)=0, \operatorname{pd}\left(b_{1} A\right)=1, \operatorname{pd}\left(a_{2} A\right)=2, \ldots$, so that $\operatorname{rfPD}(A)=\infty$.

5. Let $\Gamma_{i}$ be the quiver above using only the arrows $\left\{a_{j}, b_{j}: j \leq i\right\}$, and let $A_{i}$ be the algebra obtained by specifying relations as above. Then $A_{i}$ is a finite dimensional algebra with $\left(\operatorname{rad} A_{i}\right)^{4}=0$ and exactly two isomorphism classes of simple right $A_{i}$-modules. To compute $\operatorname{gldim}\left(A_{i}\right)$ (and show it is finite) we need only compute $\operatorname{pd}\left(\operatorname{rad} A_{i}\right)$. The computation of right annihilators in $A_{i}$ is the same as that in $A$. Note that $\operatorname{rad} A_{i}=\left(a_{1} A_{i}+\cdots+a_{i} A_{i}\right) \oplus\left(b_{1} A_{i}+\cdots+b_{i} A_{i}\right)$ and $b_{1} A_{i}+\cdots+b_{i} A_{i}=b_{1} A_{i} \oplus \cdots \oplus b_{i} A_{i}$, so $\operatorname{pd}\left(b_{1} A_{i}+\cdots+b_{i} A_{i}\right)=2 i-1$. To compute $\operatorname{pd}\left(a_{1} A_{i}+\cdots+a_{i} A_{i}\right)$ we inductively use the exact sequence,

$$
0 \rightarrow I \cap y A_{i} \rightarrow I \oplus y A_{i} \rightarrow I+y A_{i} \rightarrow 0
$$

Noting that $\operatorname{pd}\left(a_{j} A_{i}\right)=2 j-2,\left(a_{1} A_{i}+\cdots+a_{j-1} A_{i}\right) \cap a_{j} A_{i}=a_{j} b_{j} A_{i}$, and $\operatorname{pd}\left(a_{j} b_{j} A_{i}\right)=2 j-1$, we find that $\operatorname{pd}\left(a_{1} A_{i}+\cdots+a_{i} A_{i}\right)=2 i$. It follows that $\operatorname{gldim} A_{i}=2 i+1$; note that $\left[\operatorname{rad} A_{i}: k\right]=2 i^{2}+i$.

\section{REFERENCES}

[B] H. Bass, Finitistic dimension and a homological generalization of semiprimary rings, Trans. Amer. Math. Soc. 95 (1960), 466-488.

[G] E. L. Green, Remarks on projective resolutions. Representation theory II, (Proc. Second International Conf., Carleton Univ., Ottawa, Ont. 1979), Lecture Notes in Math., vol 832, Springer-Verlag, Berlin, 1980, pp. 259-279.

[GKK] E. L. Green, E. Kirkman, and J. Kuzmanovich, Finitistic dimensions of finite dimensional monomial algebras, J. Algebra (to appear).

[GZ-H] E. L. Green and B. Zimmermann-Huisgen, Finitistic dimension of Artinian rings with vanishing radical cube, Math. Zeit. (to appear).

[GHZ] E. L. Green, D. Happel, and D. Zacharia, Projective resolution over Artin algebras with zero relations, Illinois J. Math. 29 (1985), 180-190.

[Gu] W. H. Gustafson, Global dimension in serial rings, J. Algebra 97 (1985), 14-16.

[IZ] K. Igusa and D. Zacharia, Syzygy pairs in a monomial algebra, Proc. Amer. Math. Soc. (to appear).

[S] A. H. Schofield, Bounding the global dimension in terms of the dimension, Bull. London Math. Soc. 17 (1985), 393-394.

[Z1] D. Zacharia, Graded Artin algebras, rational series and bounds for homological dimensions, J. Algebra 106 (1987), 476-483. 
[Z2] D. Zacharia, Special monomial algebras of finite global dimension, Perspectives in Ring Theory, Kluwer Academic, Dordrecht, 1988, pp. 375-378.

[Z-H] B. Zimmermann-Huisgen, Bounds on finitistic and global dimension for Artinian rings with vanishing radical cube, preprint.

Department of Mathematics, Wake Forest University, Winston-Salem, North CarOLINA 27109

E-MAIL ADDRESS (CSNET), Ellen KIRKMAN: EEK@WFU.EDU

E-MAIL ADDRESS (CSNET), JAMES KuZMANOvich: JJK@WFU.EDU 